\title{
Benedictus Textor y la enseñanza de la «Materia Médica» de Dioscorides
}

\author{
José MARÍA VALDERAS
}

\section{RESUMEN}

Exhaustivo estudio de las Stirpium differentiae de Benedictus Textor (1534), obra que intenta en su época que los futuros médicos conozcan de una forma sistemática las propiedades galénicas de las plantas, recuperando de modo científico el significado exacto de la obra de Dioscórides.

\begin{abstract}
Exhaustive study of Benedictus Textor's Stirpium differentiae (1534), a work endeavouring at this time to convey systematically to students of medicine the galenic properties of plants; a scientific adaptation of the meaning of Discorides' work.
\end{abstract}

Entre los géneros más asentados en la literatura médica medieval, y que se prolonga hasta el siglo XVI, sobresale el de la compilación de referencias de distintos autores a un mismo asunto. Pandectae y Aggregationes son quizá los títulos que primero suelen venir a la memoria. Una de las expresiones verbales habituales en los escritos histórico-bibliográficos de un Conrad Gessner juvenil es la de "digessit», para indicar que este o aquel autor extractó y aunó lo expuesto por otros. A veces, la compilación de marras llevaba aparejada una clasificación más o menos elaborada y completa de los temas abordados (facultades o virtudes de los fármacos, por ejemplo, de acuerdo con la teoría galénica de los grados). Otras veces, ese afán esquemático se resolvía en meras tablas enfrentadas de enfermedades y simples o compuestos indicados, con su nivel de eficacia, o en listas de plantas ordenadas alfabéticamente en distintos idiomas (griego, latín, árabe, hebreo y lenguas vernáculas); así, en los Synonyma de 
Simon Cordo de Génova. Se transmite, por lo común, un saber ajeno, y lo máximo que se discute es la justeza o no de la interpretación.

Se sabe muy poco de la vida de Benedictus Textor. Apenas las fechas de la aparición de sus opúsculos ${ }^{1}$ :

De cancri natura et curatione, ex probatissimis quibusque autoribus, De la manière de préserver de la pestilence, et d'en guérir, selon les bons autheurs y Stirpium differentiae ex Dioscoride secundum locos communes, opus ad ipsarum plantarum cognitionem, admodum conducibile.

Nos centraremos en el último, en las Stirpium differentiae. Publicado por vez primera en 1534 (el prólogo lleva fecha de los idus de junio de ese año) y reimpreso en 1537, se añadió a modo de apéndice a la versión latina de 1552 de la obra máxima de Hieronymus Bock (Tragus) ${ }^{2}$. Esta última reimpresión, introducida a instancias de Conrad Gessner ${ }^{3}$ por David Kyber, traductor al latín de la edición alemán de la obra de Bock, es la que manejaremos aquí.

\footnotetext{
1 Durling, R. (ed.). 1967. A Catalogue of Sixteenth Century Printed Books in the National Library of Medicine. Bethesda, Maryland: National Library of Medicine. Se recogen aquí las siguientes obras de Textor: De cancri natura et curatione, ex probatissimis quibusque autoribus, tum Graecis, tum Latinis... Lugduni; Apud Joan. Tornaesium; 1550. Su traducción francesa De la nature du chancre, selon les meilleurs autheurs tant grecs que latins... Lyon, Jean de Tournes, 1550. De la manière de préserver de la pestilence, et d'en guérir, selon les bons autheurs... Lyon, Jean de Tournes, et Guil. Gazeau, 1551. Copie des lettres de l'auteur, translatecs de latin en françois... envoyées à Maistre Jean Volat... pour avoir son advis sus l'affaire de la peste: p. 17-18; Résponse (de Jean Volat)... qui est une dependence de l'oeuvre presente traduite de latin en françois: p. 19-22. Stirpium differentiae ex Dioscoride secundum locos communes, opus ad ipsarum plantarum cognitionem, admodum conducibile; Parisiis, apud Simonem Colinaeum, 1534. Reimpresa esta obra en 1537 en Venecia, en la imprenta Divi Bernadini.

${ }^{2}$ Hieronymi Tragi. De stirpium, maxime earum, quae in Germania nostra nascuntur, usitatis nomenclaturis, propriisque differentiis, neque non temperaturis ac facultatibus, Commentariorum Libri tres, Germanica primum lingua conscripti (la edición alemana apareció en el mismo editor en 1551) nunc in Latinam conversi, Interprete Davide Kybero Argentinensi. His accesserunt a fronte Praefationes duae: altera D. Conradi Gesneri Tigurini, Medici clarissimi, rei herbariae scriptorum, quae in hunc usque diem scripserunt, catalogum complectens: altera ipsius Authoris, herbariae cognitionis laudes, et alia nonnulla scitu tum necessaria, tum iucundissima, continens. Praeterea, Corollarii vice ad calcem operis, adiectus est Benedicti Textoris Segusiani de Stirpium differentiis, ex Dioscoride secundum locos communes, Libellus, omnibus plantariae cognitionis studiosis utilissimus. Ad haec Indices sex: quibus non tantum Nomenclaturae Graecae, Latinae, Germanicae, Arabicai, Hebraicaeque, sed et morborum curationes optimo ordine indicantur, subiunximus. Cautum est Priviligeio Caroli V Imperatoris, ne quis intra septenium Typographus a se impressum aedat: aut aliunde empta vendat. Argentorati. Excudebat Vuendelinus Rihelius, Anno M.D.LII.

3 "Caeterum consilio excellentissimi Medici Domini Conradi Gesneri, libellum Benedicti Textoris, cuius argumentum et usum facile ex inscriptione, et Praefatione cognosces, coronidi vice adiecimus." ("Davidis Kyberi candidum lectorem Praefatio» en HieronYmI TRAGi. De stirpium, maxime earum, quae in Germania nostra nascuntur..., s.p.
} 
Limitados por datos tan escuetos, podemos acotar la época de actividad de Textor entre 1530 y 1556. El análisis interno de la obra escrita permite adentrarnos algo más en su perfil. Discípulo de Jacques Dubois (Silvio), poseía una sólida formación humanística y echó su cuarto a espadas en uno de los temas redundantes de mediados del siglo XVI, la peste. Por lo que a la historia de la botánica concierne, su Stirpium differentiae rubrica la consagración escolar de la Materia medica del anazarbeo en la Facultad de Medicina de París en los primeros años treinta y ahorma o encarrila las descripciones de las floras y comentarios que irán apareciendo hasta el nuevo. rumbo que marca la publicación del De plantis libri XVI de Andrea Cesalpino, en los ochenta. La adscripción galenista de Silvio pesó en la elección del tema. Del corpus del pergameno se venía haciendo amplio uso el tratado sobre la dieta, De alimentorum quidem facultatibus per locos communes, cuya semejanza en el propio título con el escrito de Textor es más que llamativa. Pero éste va más allá. Su librito no se queda en lo "médico", sino que busca un apuntalamiento en la "grammatica» y la "philosophia naturalis». Atiende, pues, a la identificación de las plantas, a su naturaleza y accidentes, a las diferencias específicas.

El opúsculo ocupa, del libro de Bock, las páginas 1129 a la 1200, ambas inclusive, antecedidas por el título Stirpium differentiae / ex Dioscoride secundum locos / communes, opus ad ipsarum plantarum / cognitionem admodum conducibile. La primera referencia escrita sobre Textor, en la que se basan las alusiones posteriores, la hallamos en el prefacio de Gessner incluido en el De stirpium de Tragus, donde recuerda que es un médico erudito que publicó años atrás nuestro opúsculo y donde pondera la pulcritud y utilidad del método empleado en la síntesis de la Materia medica de Dioscórides.

El «libellus» consta de un prólogo («Benedictus Textor Segusianus candido lectori salutem dicit») y del cuerpo de la obra ( Ex Marcelli Vergilii Commentariis in ipsum Dioscoridem, ad eorum quae hic tractantur dilucidiorem intelligentiam»). Indica, pues, que se sirve de la traducción de Dioscórides al latín, realizada y comentada por Marcello Vergilio, aunque el uso por Textor de las glosas propiamente dichas será mínimo. La versión del Dioscórides que firmó Marcello Vergilio Adriani (1464-1521) apareció en Florencia en 1518, y contenía, amén de la traducción del texto, comentarios personales y «correciones" a la versión de Hermolao Barbaro, aparecida dos años antes. Textor manejaría tal vez la edición de Vergilio publicada en Basilea en 1532. Posterior a esas traducciones de Hermolao y Vergilio es la divulgadísima de Joannes Ruellius, quien toma buena nota de la labor desarrollada por ambos, aunque tampoco la suya está exenta de problemas, como se encargan de poner de manifiesto los botánicos subsiguientes (Brassavola, Matthioli y otros). Giovanni Manardo, por su lado, "corregirá» en sus famosas Cartas la de Marce- 


\section{JOSE MARIA VALDERAS}

llo y vindicará la de Hermolao ${ }^{4}$. Textor da por buena la traducción latina que emplea.

Según venía siendo muletilla corriente entre los humanistas, lamenta en el prólogo, con trazos duros, la ignorancia y el abandono en que habían caído, a lo largo de los siglos obscuros del Medievo, la medicina en general y la materia médica en particular, en las que brillaron los médicos y gobernantes de la antigüedad clásica ${ }^{5}$. Situación que puede invertirse volviendo a Galeno y a Dioscórides. Es decir, siguiendo el camino señalado por su maestro Silvio.

Textor debió de ser uno de los primeros discípulos de Jacques Dubois (14781555), cuando éste empieza a enseñar medicina en París al iniciarse la década de los treinta. Silvio, quien le encarga la redacción del opúsculo ${ }^{6}$, conocedor de la materia médica clásica y arabizada, defendía en su empeño restaurador de la anatomía, una vuelta cuasi literal al galenismo depurado, recuperación en la que ejercía un papel decisivo el conocimiento de las lenguas clásicas. La impronta de Silvio se refleja desde el comienzo. Textor ha de acometer un trabajo anatómico de los organismos vegetales, que resultará de la máxima utilidad: «ex illo authore (Dioscoride) historia per partes plantarum digesta, rursus singula membra dissecarem per locos communes, ille magno usui id fore affirmans» (Del prefacio; las cursivas son mías).

No menos importante que la vía anatómica es el criterio de agrupamiento. El quicio sobre el que descansa la partición son los «loci», un concepto básico del Organon aristotélico, cuerpo lógico que estaba sufriendo una profunda transformación desde el último tercio del siglo XV en buena parte de Europa y, desde principios del XVI, en París. Coinciden esos años con los del paso de Textor por la facultad de artes, antes de iniciar el estudio de la medicina. En esos años adolescentes, los futuros médicos, teólogos y hombres de leyes se familiarizaban con la filosofía y la ciencia aristotélicas. Estudiábanse en el París de Textor compendios lógicos menores, como las Sumulas de John Mair, prototipo de las parva logicalia, que exponían sobre todo los términos y su inclusión en las proposiciones. Se recomendaba la lectura directa de los tratados origi-

\footnotetext{
${ }^{4}$ VALDERAS, J.M., 1995. «Aproximación a las Epistolas de Giovanni Manardo». Collectanea Botanica (Barcelona): 23, en prensa.

5 "Inter tam foedas tamque monstrosas labes, quibus conspurcatus et adulteratus est aliquot iam saeculis ille Medicinae decor et splendor, ut non alta pertinacius haeret eorum medicamentorum maximeque stirpium, quae tanto studio tantoque honori veteribus non modo medicis, verum regibus quoque ipsis fuerunt, ignorantia et neglectu: ita nihil est homini medico magis pudendum.» (Praef.).

6 «Proinde quando hunc unum propemodum habemus farciende rei longae praestantissimae, ac nobilissimae tantopere commendatum, praeceptor ille noster Jacobus Sylvius praeter summam eruditionem publicae utilitatis studiosissimus saepenumero me hortatus est ex illo authore historia per partes plantarum digesta, rursus singula membra dissecarem per locos communes, ille inquam magno usui id fore affirmans.» (Praef.).
} 
nales del estagirita: Analíticos, Categorías, Sobre la interpretación y los Tópicos, así como los Físicos para la filosofía natural. Aristóteles dedica todo un libro, los Tópicos, a la cuestión de los lugares, es decir, a las fuentes de donde deben tomarse los argumentos para la demostración y discusión de los problemas científicos y filosóficos. Las categorías, desde Quintiliano, se venían considerando lugares y constituían la base idónea para las clasificaciones y ordenación del saber de cualquier disciplina. En particular, la doctrina de los loci communes fueron objeto de estudio en las «dialécticas» del humanismo parisiense, influidas sobre todo por la obra de Rodolfo Agricola (1444-1485) De inventione dialectica libri omnes et integri ${ }^{7}$.

Los listados de hierbas, sin más trabazón interna que la seriación alfabética, venían constituyendo el núcleo de la farmacopea medieval. Se fueron incrementando con nuevas especies que aportaba la recuperación de los manuscritos y traducciones grecolatinas. Las versiones latinas de Dioscórides en la segunda década del XVI acabaron por entronizar a éste en el centro de la terapia. Pero, ¿cómo sacarle el mayor partido? ¿Cómo sistematizarlo y memorizarlo? A esas cuestiones responde el propósito de Textor. Tarea ardua. Por eso, insiste en su juventud y en la dificultad del empeño, a modo de excusa para rechazar la invitación que termina por aceptar viniendo de quien viene ${ }^{8}$.

No era la primera vez que en el Renacimiento se acudía a las categorías de Aristóteles para establecer criterios de identificación y ordenación de especies. Leoniceno y Poliziano habían roturado ya ese terreno ${ }^{9}$. Silvio, no mucho después, generalizó ese tipo de trabajos para la enseñanza y para la práctica médica, y él mismo publicará cinco años más tarde sobre dicho método su Ordo, et ordinis ratio in legendis Hippocratis et Galeni libris ${ }^{10}$.

Antes de entrar de plano en el cuerpo del opúsculo, Textor introduce la definición de una serie de nociones para mejor interpretar la parte posológica y

\footnotetext{
${ }^{7}$ Colonia, 1523. Reprint: Minerva, Frankfurt, 1967.

8 "Quid quaeso tantae doctrinae viro denegarem, adeoque de omnibus benemerito? Ego itaque non ignarus quam ineptus essem ei provinciae, aliquandiu meam excusans infantiam, tandem parere sum coactus. At non multum post tanquam desperebundo (ut qui rem nullius aut parvi momenti futuram existimarem, quae alioquin magno mihi constaret) in animo fuit desistere. Ille contra: Nam, inquit, hoc adeo laeve videtur ac frivolum, cum cuiusvis proclive sit id veluti filium sequuto primo statim occursu deprehendere stirpem nec antea visam? Ita res obeunda fuit pro viribus. Quod ne ille ipse quoque sedulo obstitisset, ne aliis parum consulens idipsum pateretur apud me diletesere ceu quod emitteretur indignum, per me quidem in lucem prodiisset nunquam.» (Praef.).

9 VALDERAS, J. M. 1990: «Errores botánicos de Plinio señalados por Leoniceno». Collectanea Botanica (Barcelona) 18: 117-138. HoPPE, Brigitte. 1976. Biologie Wissenschaft von der belenten Materie von der Antike zur Neuzeit. Biologische Methodologie und Lehren von der sotffichen Zusammensetzung der Organismen. "Sudhoffs Archiv», Beinheft 17. Franz Steiner Verlag GBMH. Weisbaden).

10 Paris: C. Wechel, 1539.
} 
descriptiva de las explicaciones de.Dioscórides. Busca a veces el apoyo de un autor clásico (Plinio, Paladio) para corroborar su acepción. Empieza por lo que la física tardomedieval denominaba el capítulo de «mensuris, ponderibus et numeris»: codo es lo que va de la articulación del mismo hasta la punta del dedo medio ${ }^{11}$; palmo mayor, intervalo comprendido entre la punta del pulgar y el extremo del meñique en una mano humana extendida ${ }^{12}$; palmo menor, que equivale a la extensión de cuatro dedos consecutivos y juntos ${ }^{13}$. Sigue con la distinción entre «liquor» y «succus». Por el primero entiende el humor que brota de la parte de la planta donde se ha realizado algún tipo de corte o incisión ${ }^{14}$, en tanto que el segundo es la exudación de la planta entera, o de alguna parte de ella, troceada, machacada, prensada o cocida ${ }^{15}$.

Adelanta también algunas definiciones de términos botánicos que pudieran inducir a error al lector primerizo o poco avisado. (Como se desprende de las notas que siguen, Textor sigue agrupando todavía las plantas en los cuatro tipos principales de Teofrasto, «arbores», «frutices», «olera», «herbae», y subtipos menores: «ferulae», «legumina», «fruges», etcétera. Más adelante veremos que amplía la división cuatripartita.) Los zarcillos, por ejemplo, no son sólo los que reconocemos en la vid, sino estructuras que se repiten en otras plantas por las que éstas reptan o se encaraman en árboles y arbustos ${ }^{16}$. Llama adventicias aquellas partes supernumerarias que nacen de una principal o

11 «Cubitus, quantum a flexura cubiti usque ad summum medium digitum patet. Credimusque nos extrinsecam cubiti flexuram intelligendam» (Stirpium differentiae, p. 1131).

12 «Spithame Grajecis, Latinis authore Plinio libro septimo capite iii, dodrans, longitudo, et quod capit spatium humana manus summo pollice usque ad summum minimum digitum extensa, quae mensura quoniam in tres quartas humani pedis partes incurrit, dodrans dicta est." (Stirpium differentiae, p. 1131).

13 "Palaestes, Palmus, mensura est simul coniunctorum, et cohaerentium quatuor digitorum.» (Stirpium differentiae, p. 1131).

14 "Liquor, humor, qui secta, terabrata, castratave planta, aut aliqua eius parte quasi sanguis aperta vena perfluit." (Stirpium differentiae; p. 1131).

15 «Succus, ex tota materia, parteve eius aliqua concissa tusave, adspersis aliquando aqua vinove, aut humore alio, tum manibus tum torculari aut igne expressus exudatusque humor.» (Stirpium differentiae, p. 1131).

16 "Viticulas voco, Plinii Palladiique verborum utens, non vitium palmites et sarmenta, sed ex eorum similitudine in aliis plantis veluti flagella, qualia in cucurbitis, cucumeribusque cunctis passim videntur per terram iacentia, aut si prope arbores et frutices sint, eos scandentia, suisque claviculis quasi manibus vitium modo haerentia." (Stirpium differentiae, p. 1131).

17 «Sunt nobis adnata et adnascentia, quae graecis paraphyades, quod adnascantur, et super accedant, sic appellatae quae dicuntur eodem modo in caulibus et ramis plantarum super terram, et in radicibus aliquot sub terra, a principio suo, veluti matre quadam, nova germinatione prodeuntia. qui naturae et nascendi ordo in bulbosis radicibus plerumque est, et in Lilio maxime, una radice, ut ait Plinius, quinquagenos saepe remittente bulbos. Sunt autem ea olerum et herbarum propia, quemadmodum in arboribus privatim, rami appellantur.» (Stirpium differentiae, p. 1131). 
"madre» ${ }^{17}$. De las axilas surge siempre un rebrote; tienen, pues, interés morfogenético. Lo que no ocurre con las partes adventicias, que carecen de lugar seguro donde nacer; en la explicación que da asoma el patrón anatómico subyacente que le guía ${ }^{18}$. Los zarcillos le llevan, por asociación, a los capréolos $^{19}$ de las trepadoras. Supone impropiamente que no es flor el nucamento, flor masculina de ciertos árboles ${ }^{20}$. Ignorábase todavía la naturaleza floral y función transmisora del vilano ${ }^{21}$.

Textor se apresta, sin embargo, a perfilar el significado exacto del término fruto y sus formas (pomo, legumbre), al tiempo que refleja el sentido finalista de la naturaleza, de raíz aristotélica: el fruto es para propagar las semillas ${ }^{22}$. Le interesa, por encima de todo, delimitar la equivalencia exacta de los términos griegos empleados por el escritor (Dioscórides). Dentro de ese afán esclarecedor debe entenderse la inclusión del epifilo de la vid, "concebido en último lugar, inútil y despreciado casi siempre en la vendimia» ${ }^{23}$. Y ciertas formacio-

18 «Graeci translata ab humano corpore appellatione majalas dicunt cava, quae aliquando bina, aliquando singularia, et ex intervallo semper in plantis videntur humanis alis similia, in quibus veluti lacuna est. Sunt autem homini alae qua parte brachium humero coniungitur ex interiore parte, in quibus non aliter quam in pectine pubes aliqua nascitur virosi plerunque odoris. Earum similitudine concavos geniculos, quales in harundinibus et plerisque aliis sunt, majalas Graeci, Romani alas dixerunt: ab adnatis eo differentes, quod ex alis nova semper germinatio prodit, adnata vero nullam habent certam partem unde emergant.» (Stirpium differentiae, p. 1131).

19 «Helix apo tou eliesthai, id est, a circumagendo et involvendo se, Latine a capiendo capraeolus, item claviculus dicitur. Sunt autem claviculi, sive claviculae viticularum in plantis extremae partes et colliculi intorti quibus advolvendi et implicandi se propinquis stirpibus natura est.» (Stirpium differentiae, p. 1132).

20 "Nucamentum in quibusdam arboribus pro flore est, veluti in piceis, pinis et corylis. Quale autem id sit quotannis licebit observantibus videre.» (Stirpium differentiae, p. 1132).

21 "Sunt Graecis et Latinis pappi lanosae villositates caudarum in animalibus forma, quae decidentibus floribus in plantis aliquot remanent, ut in utraque Soncho, in Carduis multisque aliis fit: quos solent aliquando pueri ludentes, spiritu oris per aerem pellere levitate sua volucres et quantumvis parvo veluti flabro diffluentes. His etiam ad ventorum tempestatumque praesagia in agro agentes quasi certissimis nuntiis utuntur." (Stirpium differentiae, p. 1132).

22 «Karpós, id est, fructus etsi aliquando in scriptore hoc semen significat, propria tamen eius significatio est in his quae causa seminis a natura fiunt. Verbi causa in pomis proprie semina dicitur interiora grana, karpós autem tota cum seminibus poma declarat, id est, claudentem ea exteriorem illam carnem. In leguminibus itidem semina, quae siliquis clauduntur, karpós vero, id est fructus cum seminibus integras siliquas. Et ne longiores fimus quodcumque seminis causa factum est, id proprie karpós Graeci vocant. Miscentur tamen haec quoniam alterum alterius causa est. Nec male penitus karpon fructum scilicet aliquis semen appelllet, veruntamen in aliquibus distingui ea oportet.» (Stirpium differentiae, p. 1132).

23 «Epiphyllis a Graecis dicitur ultimus in summo vitis palmite racemus, qui plerunque parvus rarisque acinis sero maturescit, quoniam postremus conceptus est, ideoque inutilis et in vindemia plerunque neglectus.» (Stirpium differentiae, p. 1132). 


\section{JOSE MARIA VALDERAS}

nes espúreas ${ }^{24}$. Persiste en la confusión en torno al perianto ${ }^{25}$. La relación entre flor y fruto en la rosa le conduce a aclarar otra asociación llamativa, que presenta en Dioscórides una denominación propia: los citinos $^{26}$. A propósito de la flor, cree necesario exponer el origen y morfología de la umbela y dar ejemplos de umbeláceas 27 ; a propósito del fruto, atribuye al pericarpio, que es lo que envuelve al karpós y difiere de unas plantas a otras, una función protectora contra la inclemencia del tiempo o la agresión de los animales ${ }^{28}$.

Tras explicar al lector el significado genuino de algunas partes de la planta para Dioscórides, aborda otras que el anazarbeo describe por comparación ("De iis quae hic fit collatio»). Textor se limita, en su opúsculo, a ordenar lo que se lee en la Materia medica. En la comparación, se da por descontado que las especies son inamovibles y de igual rango. Las diferencias y las semejanzas (la distancia o la proximidad) remiten a notas que se predican de unas especies con las que se supone que el lector se halla más familiarizado, sin que denuncien, en ningún momento, una relación de subordinación o preeminencia, de jerarquía. Conviene, sin embargo, conocer bien los términos de la comparación. Así ocurre, por ejemplo, con la hoja del llantén mayor (Plantago major L.) que se parece a la de las hortalizas, pero no a todas - pues no existe una hoja común característica-, sino a las de hoja más ancha, como la de la acelga (Beta vulgaris L. ${ }^{29}$. Esta acotación de Textor se mantendrá en los comentarios y apostillas que irán apareciendo en las reimpresiones de la traducción del

\footnotetext{
24 «Cachrys pilula est, quam arbores aliquot praeter proprium fructum proferunt. Hanc poterit aliquis,si alias non liceat contemplari arbores, in Pino et Iuglande nuce quotannis per hyemem videre. Caret Latino nomine Cachrys haec, quemadmodum et Libanotidis semen, quod interposita una litera Canchryn Graeci vocant.» (Stirpium differentiae, p. 1132.).

25 «Dixerunt in floribus antiqui calyces involucra illa qualia in rosis sunt, quibus dehiscentibus in sua folia flores pandunt." (Stirpium differentiae, p. 1132).

26 "Cytini flores sunt punicae a tumore Graecis appelati, qui kyton, quod ambitu et tumore suo aliquid capit et in se claudit, dixerunt.Nam Cytini captu et ambitu suo futurum pomum iam tunc intra se continent, et quasi conceptos acinorum foetus ad maturitatem complectuntur.» (Stirpium differentiae, p. 1132-1133).

27 «Umbellam Latini, skiadion Graeci, ab umbra utrique appellant in aliquibus plantis floris veluti pedamentum in plures divisum longiores pediculos, qui singuli florem sustinent et in orbem circinnatum: qua floris figura Foeniculum, Anisum, Sambucus, pluraque alia sunt. Quod quoniam umbellae, qua solem a vultu mulieres arcent, forma est ob latitudinem circinationemque suam, ideo Graeci primum eam formam skiadion, mox Graecos imitati Latini Umbellam dixerunt.» (Stirpium differentiae, p. 1133).

28 «Sunt Graecis pericarpia cuncta illa quae semen aliquod ambientia a coeli et animalium iniuriis id servant et tuentur. Quod licet in leguminibus privatim siliqua, in frugibus spica, in casteneis echinus sit, in aliis vero, aliis appellationibus indicentur, communi tamen nomine cuncta haec pericarpia dicuntur.» (Stirpium differentiae, p. 1133).

29 "Maioris plantaginis folium lajanodis, id est, oleraceum dicitur, non tamen simpliciter ad omnia olera id referendum est, falsum enim id esset, sed ad ea tantum quae latiore sunt folio, qualis Brassica et Beta est.» (Stirpium differentiae, p. 1133).
} 
Dioscórides (en la de Ruelle, por ejemplo, y nuestro Laguna). Otra hoja singular de no inmediata intelección en Dioscórides es la de lámina larga y limbo redondeado, a modo de sombrero, petasatus ${ }^{30}$; a veces se aplicaba también esa expresión a la forma de ciertos hongos. Traído por la semejanza de forma, del sombrerillo al ombligo de Venus (Umbilicus pendulinus DC), Textor introduce dicha planta, cotiledón ${ }^{31}$, aprovechando la oportunidad para desarrollar la evolución histórica, basada en Plinio, de la medida de volumen del mismo nombre. Al hilo de la traslación histórica de significado de otros términos, muestra el origen de la denominación de ciertas plantas. El acanto ${ }^{32}$ (Acanthus spinosus L.), el ricino ${ }^{33}$ (Ricinus communis L.) y el fruto del castaño ${ }^{34}$ (Castanea sativa Miller).

Concluido ese apartado introductorio, Textor acomoda el Dioscórides al cuadro de las categorías aristotélicas para establecer las diferencias entre las plantas, primero en razón de su naturaleza y después en razón de sus partes integrantes, de acuerdo con los lugares comunes. Para Aristóteles, las categorías designan las maneras múltiples que tiene el ser de significar. Las categorías son determinaciones del ser. Frente a la decena acostumbrada, Textor emplea las siete siguientes: sustancia, cantidad, cualidad, sitio (dónde), tiempo (cuán-

\footnotetext{
30 «Petasus, i. Galerus graeca vox est quae Mercurii et Augusti Caesaris usu in antiquis scriptoribus celebratur. Uterque enim petasatus describitur, sed alter dum coelo praecipitem se mittit avi similis: alter dum per diem in sole est, radiosque eius munito capite, petaso hoc, toto anno vitat.» (Stirpium differentiae, p. 1133).

31 «Cotyledon facta est a cotyla Graecorum in poculis et convivio mensura vini et vasculo, quam Plinius aliquando heminam, nonum quam sextariam ex Graecis vertit. Eius forma erat qualis nunc etiam in fictilibus poculis videtur, quibus in paupertate et cenobiis utuntur, et cotylam nostra gens ad huc vocat. Fuitque hoc eius nomen primum semperque in poculis et liquidorum mensura. Quoties autem servata eiusdem vasculi forma in alium transferebatur usum, ocypbaphum vocabatur, quod in coena aceto plenum ad instinctus ponebatur. Et haec graece cotylae et oxybaphi prima ratio et usus fuit. Romani in priore illo poculorum usu, et liquidorum mensura sextario, et hemina usi sunt. Cum vero ad intinctus uterentur, ab aceto acetabulum dixerunt, cuiusforma orbicularis esset, profunda et sine marginis latitudine. Ab his deinde utraque gens suum nomen ad alia, quae similem haberent concavitatem, transtulit, in quorum numero est Cotyledon herba.» (Stirpium differentiae, p. 1133).

32 «Spondylia Graecis sunt in animalium spina vertebrae seu nodi eodem nomine dicti, quo mulieres in fusis suis verticillos nominant, quibus in ducendo filo graviore eorum pondere suos librant.» (Stirpium differentiae, p. 1133-1134).

33 «Sunt latinis ricini et Graecis krotonie insecta quae animalibus aliis canibusque praesertim inhaerent sine motu cum semel haeserint. Quibus cibi nullus exitus est, colore livido, nullis discretis membris, in globum continue crescentia, donec plenius saginata post aliquos dies sponte sua decidant." (Stirpium differentiae, p.1134).

34 «Echinus marinus ex testaceorum genere piscis dicitur, cui natura est spinis quasi pedibus et earum in orbem volutatione ingredi. Quem facile noverit is qui in castanea summum fructus eius aculeatum, et in orbem circumvolutum operimentum viderit.» (Stirpium differentiae, p. 1134).
} 


\section{JOSE MARIA VALDERAS}

do), acción y uso. Aunque Aristóteles no habla de la categoría «uso», sabido es que tampoco da por cerrado el número de categorías; además, podemos asociar. el «uso» con la categoría «pasión».

En las plantas, las categorías tienen sus propias determinaciones. Para Textor, hablando del vegetal entero o de sus partes, la cantidad se expresa a través del número y del tamaño; la cualidad, a través del aspecto visible (brillo, color), del olor, del sabor, sensibilidad al tacto, figura y semejanza (con otro o con varios más); el sitio, a través del lugar donde medra; el tiempo, a través de su nacimiento, muerte y maduración; la acción, a través de su eficacia médica; el uso, a través de su destino (médico, ornamental, etcétera) ${ }^{35}$. Textor concede particular atención a la categoría "semejanza», que podemos asociar a la aristotélica relación ${ }^{36}$.

La consideración de las plantas en razón de su naturaleza vegetal, es decir, en razón de las distintas notas con las que Dioscórides va determinándola a lo largo de su descripción («plantarum discrimen a toto acceptum»), abarca de la página 1134 a la 1136. El principal rasgo discriminante es el porte o la talla. En razón del mismo, Textor entiende que Dioscórides dobla el número de los tres primeros grupos teofrasteos (árboles, arbustos y matas): «arbores», «arbusculi», "frutices», "plantae arborescentes», "herbae fruticosae» y «herbae similiores arboribus». Las tres primeras más próximas a la naturaleza de árbol o arbusto y las segundas a las de arbustos también o matas. Textor se limita, en efecto, a ordenar lo que dispersamente escribe Dioscórides. Pero lo que en éste no tenía más que un mero carácter descriptivo, en Textor adquiere categoría de grupo, donde debe insertarse la especie en cuestión. En la déscripción de cada uno de los distintos ejemplos extraídos, Dioscórides, en la versión de Marcello Vergilio, señala verbatim el carácter del porte (arbóreo, arbustivo, etcétera); Textor lo hipostatiza.

\footnotetext{
35 Los términos empleados por Textor son Substantia; Quantitas (numerus, magnitudo); Qualitas, qualitas visilis (fulgor, color), odor, sapor, tactilis qualitas, figura, similitudo (unius, duorum aut plurium); situs; tempus (ortus, occasus, maturitas): actio; usus.

36 "Se dicen respecto a algo todas aquellas cosas tales que, lo que son exactamente ellas mismas, se dice que lo son de otras cosas o respecto a otra cosa de cualquier otra manera; v.g.: lo mayor, aquello que es exactamente, se dice que lo es comparado con otro, pues se dice doble de alguna cosa; de la misma manera también todas las demás cosas de este tipo. También son de lo respecto a algo cosas como éstas: estado, disposición, sensación, conocimiento, posición; en efecto, todas las cosas mencionadas, lo que son exactamente ellas mismas, se dice que lo son de otras, y nada más; en efecto, el estado se llama estado de algo, y el conocimiento, conocimiento de algo, y la posición, posición de algo; y de la misma manera el resto. Así, pues, son respecto a algo todas aquellas cosas que, lo que son exactamente ellas mismas, se dice que lo son de otras, o respecto a otra de cualquier otra manera; v.g.: una montaña se llama grande respecto a otra cosa —n efecto, la montaña se llama grande respecto a algo-, y lo semejante se dice semejante a algo, y las demás cosas de este tipo se dicen de igual manera.» (Categorías 7 6a-6b).
} 
Ejemplos de los «arbores" extraídos de la Materia medica son: «Ricinus» (Ricinus communis L.), "Smilax quae Latinis Taxus dicitur» (Taxus baccata L.), "Cornus» (Cornus mas L.), «Oxycantha» (Cotoneaster pyracantha Spach.), "Sabina» (Juniperus sabina L.), "Tilia» (Phillyrea angustifolia L.), "Lycium» (Rhamnus infectoria L.), "Arbutus" (Arbutus unedo L.), "Sycomorus» (Ficus sycomorus L.). De entre las «arbusculae» dioscorídeas señala el "Rhù (Rhus coriaria L.), el zumaque que Laguna prefiere denominar mata, y la «Sabina altera» (Sabina cupressus L.). Por último, «Fruticosa arbor, Erica» (Erica arborea L.), un árbol ramoso, así como el tamarisco, aunque harto menor, en palabras de Laguna ${ }^{37}$.

Lo que Dioscórides, Vergilio mediante, llama «Arborescentes plantae» suelen ser arbustos o matas que miden por lo común entre casi dos y tres metros. Textor selecciona los siguientes: "Cynosbatos, id est, Sentis canina (Rosa canina L.) "Agnos Latinis vitex» (Vitex agnus castus L.), «Sambucus» (Sambucus ebulus L.), "Abrotonum foemina» (Santolina chamaecyparissus L.), "Anagyris» (Anagyris foetida L.), "Oenagra» (Epilopium angustifolium L.), Sonchi, id est, Cicerbitae tertium genus (ignoro a qué tipo de cerraja alude Dioscórides que, como traduce Laguna, crece en altura de árbol, salvo que, guiados por la significación del tercer tipo de calabacín designe la colquíntida, Citrullus colocynthis Schrad.), «Tithymalus sextus, unde Dendrodes, id est, arborescens cognominatur» (Euphorbia dendroides L.) ${ }^{38}$.

Rastrea numerosos ejemplos de plantas que componen el grupo dioscorídeo de las «Herbae fruticosae», aquellas que a duras penas arriban al metro de altura y suelen presentar el tallo ramoso: "Sion» (Sion latifolium L.), «Seriphium sive Absinthium marinum» (pese a la denominación, la descripción dioscorídea no respalda que se trate, como suele creerse, de la Artemisia maritima L.), «Sticas" (Lavandula stoechas L.), "Maron» (Teucrium marum L.), "Acinus» (Ocimumpilosum Willd.), "Baccharis» (Helichrysum sanguineum Boiss.), "Sefeli Creticum seu Tordylon» (Tordylium maximum L.), "Myagron» (Chamaelina sativa Crantz), "Artemisia» (Artemisia maritima L.), "Capnos, id est, fumus, seu fumaria herba" (Fumaria officinalis L.), "Botrys» (Chaenopodium botrys L.), "Chrysanthemon" (Helichrysum orientale L.), "Chamaepitys secunda» (Aiuga sp.), «Eupatorium» (Agrimonia eupatoria L.), "Chritmon Romanis Batis vocatur» (Chritmum maritimum L.) ${ }^{39}$ En el último extremo, las hierbas con pujos de árbol. Si el saúco es un arbolillo, el yezgo es hierba vivaz que quiere también

\footnotetext{
37 Laguna, Andrés de. 1555: Pedacio Dioscórides Anazarbeo, acerca de la Materia Medicinal, $y$ de los venenos mortiferos. Traduzido de lengua Griega, en la vulgar Castellana, e illustrado con claras y substanciales Annotationes, y con las figuras de innumeras plantas exquisitas y raras, por el Doctor... En Anvers, En casa de Iuan Latio. Edición facsimilar, Madrid, 1991. I, 97.

38 Stirpium differentiae, p. 1134.

39 Stirpium differentiae, p. 1134-1135.
} 
elevarse. Textor toma a ésta, el Sambucus ebulus L., como representante de las «herbae similiores arboribus» ${ }^{40}$.

Hasta aquí, los principales grupos que pueden espigarse de la lectura de la Materia medica. Pero Dioscórides atribuye otras características distintivas a las especies que va desgranando. La ordenación de las mismas permite a Textor crear unas agrupaciones secundarias. Esos criterios de ordenación son la superficie espinosa o tomentosa, el contenido en fluidos, la densidad, el color, el olor, el sabor, la textura, la belleza y la leñosidad. Dentro de cada criterio existe una gama de posibilidades, hacia arriba y hacia abajo desde un término medio. A propósito del revestimiento superficial, por ejemplo, habrá especies aculeadas (por citar uno de los muchos ejemplos aducidos, el «acanthus sylvestris», o Acanthus spinosus L.), de espinas cortas («Rubus Idaeus», o Rubus idaeus L.), hirsutas («Marrubium», Marrubium vulgare L.), con escamitas («Polypodium», Polypodium vulgare L.), lanuginosas («Ranunculi alterum genus», Ranunculus lanuginosus L.), con mucha y fina lana ("Poterion», Astragalus poterium Labill.), con pelos («Muscus marinus», Ulva lactuca L. y otras algas), musgosa («Phyllon Thelygonon», Mercurialis tomentosa L.). Son plantas que no tienen ninguna relación entre sí. Y resulta muy discutible, en algunos casos, la propiedad de ciertas inclusiones en una misma secuencia. Pero a Textor lo único que parece interesarle al establecer esa jerarquía es el indicar una pista de reconocimiento a quien se encuentre por vez primera con ellas. También le facilita al alumno la memorización a la hora de tener que describirla en los ejercicios escolares.

Dejó sentada antes la distinción entre «liquor" y "succus». Ahora, agrupará las plantas en razón de la jugosidad; si poseen algún tipo de fluido, graduará los vegetales según el testimonio de Dioscórides. "Lathyris» (Euphorbia lathyris L.) es planta que toda ella mana leche, como «Peplos» (Eurphorbia peplus L.), otras euforbiáceas y el sicómoro. De color anarajando es el humor que se extrae de la «Hirundinaria maior» (Chelidonium majus L.); amarillento, el de «Glaucium" (Galucium corniculatum Curtis.).

Lógicamente, estas agrupaciones secundarias no subdividen la clasificación principal, o por el porte. Una misma planta puede entrar aquí en varias agrupaciones. El criterio, aunque no es taxonómico, incide en la definición de la planta. Son rasgos distintivos que Dioscórides ha introducido en la reseña del vegetal, y para Textor encierran un valor diacrítico. De ahí el interés que pone en jerarquizar las notas características de la planta. El abanico a veces es estrecho, como en lo que Dioscórides llama densidad. Desde las especies espesas, que crecen en todos los sentidos, como «Lappula minor» (Euphorbia acanthothamnos Heldr. et Sart.), hasta las que se abren en anchura, como la "Sabina

40 «Herbae quam arbori similior est Chamaeacte quasi dicas sambucum humilem, Latini Ebulum appellant.» (Stirpium differentiae, p. 1135). 
altera» (Juniperus phoenicea L.), pasando por la gracilidad del «Polium montanum» (Teucrium polium L.). Otras veces el abanico es muy extenso, como el del color. Este puede ser de toda la planta, del tallo o de su interior, de su humor, porque Textor entiende que éste es un exudado de la propia naturaleza, una, del vegetal. Plantas blancas, "candidae» ${ }^{41}$, son la "Mandragora mas" (Mandragoras vernalis Bertolini), y el "Ornitogalon» (Ornitgogalum umbellatum L.), entre otras que menciona. Exudan humor de leche las euforbiáceas y el sicómoro. Tiran a blanco, alguna euforbiácea y el marrubio. Amarillea el "Botrys», la biengranada (Chenopodium botrys L.). Rezuma un fluido anaranjado "Chelidonium majus» (Chelidonium majus L.). Hierba muy roja es «Alypon» (verosímilmente un titímalo, y no la Globularia alypum, por la que se tiene). Del color de la granada es "Ascyron" (Hypericum perfoliatum L.). Enrojece las manos cuando se la coge en flor la «Ruta sylvestris» (Ruda montana L.). Presenta un verde persistente «Chamaepeuce» (Tussilago farfara L.). Negra, por fin, la «Mandragora foemina" (Mandragora autumnalis Bertolini.).

Distintos son los grados del olor que despiden las plantas. Textor recoge la expresión matizada por Dioscórides y reúne en torno a la misma las especies que participan de ese mismo grado. Hay «stirpes odorate», «odoratissimae», "tetri odoris», «graviter olens», «ingratus odor», «gravissime olens» y «aromatice olens». Por recordar un ejemplo significativo de cada subgrupo: de un suave olor, como debe entenderse aquí las «odoratae», es «Coris» (Hypericum coris L.); de un olor suavísimo, "odoratissima", "Sampsuchum" (Majorana hortensis Moench.); de un olor intenso, "tetri odoris», la matricaria o "Parthenium" (Chrysanthemum parthenium Bernhardi); de un olor muy grave, "Chelidonium maius" (Chelidonium majus L.); un tanto ingrato es el olor de "Abrotonum foemina" (Santolina chamaecyparissus L.); hediondo, "gravissime olens", es la «Anagyris» (Anagyris foetida L.); por último produce un suavísimo olor al mascarse, «aromatice olens», el "Daucus secundae speciei» (Athamanta cretensis L.).

Por su relación con la terapéutica, Textor se muestra particularmente detallista a la hora de amontonar sucesivos grupos homogéneos que tengan que ver con el sabor, con la astringencia, que "aprietan» como castizamente traslada Laguna. En este apartado, vincula las especies, siempre de acuerdo con el calificativo que le merecen a Dioscórides, en "adstringentes", "egregie astringit", «acerba», grato sapore», «dulce», «salsae», «amarae», "amaritudine quadam», "amari amarius», "acres et fervidae», «efficacissimae acrimoniae», "amara et subacris" y "acri et subamaro succo". Por traer a colación algunas muestras: entre las «adstringentes», la "Hemionitis» (Scolopendrium hemionitis Lag.); de grato sabor, o sabrosa, la "Coris" (Hypericum crispum L.); salada, el "Empetron" (Frankenia hirsuta L.), etcétera. No le importa repetir ejemplos que han

${ }^{41}$ Stirpium differentiae, p. 1135. 
aparecido ya en grupos precedentes, en el color o el olor, ni parece guiarse siempre por resaltar las que sean más familiares al alumno, al médico o al herborista. Textor se limita a diseccionar la Materia medica, catalogando su contenido de acuerdo con las categorías aristotélicas.

La textura es otra propiedad que sirve para definir la naturaleza de la planta. En virtud de la misma, según la versión de Marcello Vergilio, Dioscórides habla de plantas «molles», "tenerae», "egregiae teneritudinis», "pingues», "durae», «fortes», «cum livore», «inaequales», «scabrae», «cum asperitate aliqua» $\mathrm{y}$ "cum tenaci nexu». Blanda como el llantén es «Rumex agrestis» (Rumex aquaticus L.); tierna el "Chrysanthemon" (Anthemis tinctoria L.); muy tierna, "Capnos» (Fumaria officinalis L.); pingüe o grasa, «Sion» (Nasturtium officinale R. Br.); dura, "Paliurus» (Paliurus spina Christi Mill.); fuerte o muy dura, "Cornus» (Cornus mas L.); fuerte o "cum livore», «Dictamnus» (Origanus dictamnus L.); muy desigual, «Rhodia radix» (Sedum roseum Scop.); áspera, «Daucus secundus» (Daucus carota L. var. sylvestris); un tanto áspera, "Alyson» (Biscutella sp.); con ramos muy juntos, «Aparina» (Gallium aparine L.). Dioscórides destaca a veces la hermosura como un rasgo peculiar. Textor enumera sus grados. Hay plantas delicadas, como la "Artemisia altera» (Artemisia campestris L.), de notable elegancia, como el «Tragoriganum» (Thymus tragoriganum L.), aptos para confeccionar guirnaldas, como el «Serpyllum hortense» (Thymus serpyllum L.), y aguzados como el «Acuminatus Iuncus» (Iuncus acutus L.).

Por fin, en las descripciones dioscorídeas se alude también a una nota discriminante muy útil para el reconocimiento de una planta, la textura o leñosidad. Enteramente surculoso es el «Symphytum petraeum» (Symphytum tuberosum L.); con cabellera el equiseto (Equisetum pratense L.), y retuércese como un sarmiento el "Amomum» (Amomum cardamomum L.).

A modo de recursos mnemotécnicos, que permitan retener mejor el contenido de la Materia medica, y ayuden a asociar unas plantas con otras, o a distinguirlas, introduce un apartado que se diría sobrante si no fuera porque los destinatarios empezaban ya a manejar listados de plantas que se añadían al final de las traducciones y arribaban, desde Italia, las enmiendas («castigationes») del significado de ciertos términos. En ese contexto hay que entender el epígrafe dedicado a la relación de semejanza y disparidad entre las plantas de acuerdo con el orden que se dan en su secuencia griega ${ }^{42}$.

Junto a la palabra griega agrega la traducción latina cuando difieren, casi siempre, en su raíz. No es un diccionario exhaustivo, ni mucho menos: «Abrotoni», «Agrosteos. Graminis», «Anchusae»; "Acalyphes. Urticae», «Acanthi», "Actes. Sambuci», «Amarici», «Andrachnes. Portulacae», «Anemones»; «Anisi», «Arnoglossi. Plantaginis», «Achradis. Piri agrestis», «Absinthium», «Bati. Rubi»,

\footnotetext{
42 "Plantarum inter se similitudo et diversitas eo ordine qui in elementis Graecis cernitur." Stirpium differentiae, p. 1136. Su desarrollo cubre hasta la página 1140.
} 
BENEDICTUS TEXTOR Y LA ENSEÑANZA DE LA «MATERIA MEDICA»

«Batrachii. Ranunculi», «Glyconis. Pulegii», "Dauci. Pastinacae agrestis», «Daphnoidis. Lauroeolae», «Delphinii», «Elates. Abietis», «Elaeae. Oleae», "Helxine. Muralis», «Epithymi», «Herpylli. Serpylli», «Hedyosmi. Mentae», "Thridacis. Lactucae», "Thymi», «Isatidis. Glastii», "Calami. Harundinis», "Cichorii. Ambubeiae, Intybi agrestis», "Cissi. Hederae», "Cnici», "Conyzae. Pulicariae herbae», "Corii. Coriandri», "Costi», "Cydoniae. Cotoneae mali», "Cyparissi. Cupressi», "Cypri. Ligustri», "Libanotidis. Rosmarini», "Lichnidis», «Mali», «Meconis. Papaveris», «Myrices. Tamaricis», «Narthecis. Ferulae», "Nymphaeae», "Origani», "Pepli», «Pityos. Piceae», «Polii», «Prasii. Marrubii», "Ptereos. Filicis», "Rhamni», "Rhodi. Rosae», "Selini. Apii», "Sicyoshemeri. Cucumeris sativi», "Sisymbrii», "Scolymi. Cardui», "Syces. Ficus», "Triphylli. Trifolii», «Hyperyci», «Hyssopi», «Phaci. Lentis», "Phlomi. Verbasci», "Phylli. Folii herbae», "Chamaelonis», "Chamaedryos. Trixaginis», "Chamaepityos. Aiugae», "Ocimi». En esta sección, Textor entresaca aquellas referencias dioscorídeas que ligan a dos plantas, por distintas razones. A veces, porque la que describe es afín a otra ya comentada o más conocida y de la que difiere por otros rasgos (tamaño, longitud o anchura de las hojas, vellosidad, olor, etcétera); otras, porque son estrechamente semejantes (verbigracia, la variedad silvestre y la doméstica); o guardan cierto parecido morfológico; o porque ambas son trepadoras (como la clemátide y la hiedra); otras veces, porque suponiéndose un mismo género, la especie cultivada se comporta de forma completamente distinta de la especie silvestre (lo que considera "sérpol silvestre» difiere del doméstico, nuestro Thymus serpyllum L.).

Con el apartado sobre la comparación binaria de plantas termina la sección consagrada a las diferencias de las plantas consideradas globalmente ${ }^{43}$. Aquí los parágrafos, indicados por letra capital en negritas, llevan los encabezamientos siguientes: "Candidius est...», «Plantae apricis locis...», «Vere prodeunt...»y "Coronariae herbae». En el primer caso, amén de comparar la blancura de dos especies, se toman otros parámetros que, en el Dioscórides, vinculan una planta con otra o con varias más: semejanzas, diferencias. En el segundo caso, el nexo descansa en el lugar donde medran: plantas que aparecen en lugares asoleados («apricis locis»), principalmente, umbríos, edificados, castigados por el viento, nivosos, clivosos, en los collados, montes, cumbres, promontorios, sobre pedregales, en campos agrestes, en sembrados, en suelos jugosos y estériles, en arenales, arboledas, en cenagales, en sitios encharcados, en riberas y litorales, en las islas, plantas ruderales, en paredes y tejados, y en otros muchos asimilados o variantes. En el tercer caso, Textor selecciona de la Materia medica plantas que germinan en una misma estación, vale decir, en primavera o verano. Con la misma falta de ilación que media entre el primero y los dos casos cen-

\footnotetext{
43 «Ad binas plantas collatio». Stirpium differentiae, p. 1140. Se prolonga hasta la página 1144.
} 
trales, el cuarto agrupa las especies "coronarias», es decir, aquellas que en tiempos de Dioscórides servían para tejer guirnaldas.

Terminada la ordenación de la Materia medica en lo concerniente a las plantas consideradas en sí mismas, en su naturaleza específica, Textor aplicará el mismo patrón general, el mismo criterio de lugares comunes, a las partes componentes del vegetal. Más arriba había introducido algunas partes que no eran principales; en lo que sigue ajustará la plantilla de las categorías aristotélicas a las partes principales. Estas partes "praecipuae" son las que repasa Dioscórides en la mayoría de las descripciones de los vegetales, a saber: la raíz, el tallo, la corteza, las ramas, las hojas, la flor, el fruto y las semillas ${ }^{44}$.

Tomemos como ejemplo de esa pauta aludida el primer órgano considerado, la raíz. Se trata de poner de relieve las diferencias entre las plantas en razón de ese órgano («parte principal»). De acuerdo con las descripciones de Dioscórides: si atendemos a su superficie, hay raíces adnascentes, desnudas y pilosas; si a la forma, en tubérculo, con extrusiones, fistulosas o cóncavas; si al líquido o jugo que segregan: musgosa, jugosa y rebosante. Dentro del mismo cuadro, viene un apartado notable, el del número: raíces numerosas, únicas, dobles, triples, quíntuples o séxtuples, únicas por la zona distal y múltiples por la proximal. Número que con la talla pertenecen a la categoría de cantidad. Por el tamaño las raíces son grandes, bastante grandes, exiguas, supernumerarias, contraídas, gruesas, del grosor de un dedo o casi, de un pulgar, de un meñique, de un bastón, de corteza ancha, gráciles, de líber (que abarca también el cambium) tenue o grueso, largas, oblongas, larguísimas, de tres dedos, de cuatro, dodrantales o más, de un codo o de un múltiplo de codo, anchas.

Por lo que se refiere a plantas que pueden asimilarse entre sí por razón de la cátegoría cualidad aplicada a la raíz, Textor confecciona varios conjuntos de especies, por supuesto no disjuntos, que tienen en común el color, el sabor y demás percepciones de los sentidos. Así, agrupa plantas sin ningún lazo taxonómico que poseen blanca la raíz, las que segregan un fluido lechoso o son blanquecinas. Hace lo propio con las de raíz rubia, azafranada, amarilla, rubescente, verdosa, negra, roja, roja sólo la corteza, negra por fuera, etcétera.

Espiga luego las plantas que, de acuerdo con Dioscórides, emiten un olor. Y junta, en grupos consecutivos, las especies de olor agradable ${ }^{45}$, las olorosas, de olor fuerte, intenso, intensísimo, fresco, con olor a tierra o a vino. Acomete parejas agrupaciones de plantas en razón del sabor, de desarrollo mucho más detallado por la importancia mencionada en la terapia humoral. De acuerdo con ese rasgo, enumera plantas de raíz astringente, amarga, sosa, insípida, sua-

\footnotetext{
44 «Stirpium partes praecipuae. Radix, Caulis, Cortex, Ramus, Folium, Flos, Fructus, Semen.» (Stirpium differentiae, p. 1144. El desarrollo de las mismas abarcará hasta el final de la obra.

45 «Radice iucundi odoris». (Stirpium differentiae, p. 1147).
} 
ve al gusto, dulce, salada, picante, acerba, con variantes y compuestos ${ }^{46}$. Pertenecen a la categoría "cualidad», aplicada a la raíz de una planta, cuanto se diga sobre sobre su blandura o dureza, lo que Textor resume diciendo «tactilis qualitas». En razón de ese criterio, crea diversos grupos de especies: los de raíz blanda, los de raíz tierna, grasienta, glutinosa, dura, tenaz, áspera, pesada, con variantes y compuestos. Por lo que se refiere a la figura, distingue a partir de Dioscórides plantas con raíz firme, redonda, tubular, terminada en punta, nudosa, en tubérculo, multífida (esta última figura le da pie para incluir grupos de las que poseen pelos y cirros), testiculares, en cola de escorpión, capiliforme, juncosa, en pomo, en manzana, amembrillada, piriforme, en aceituna, en bellota, etcétera. Termina con algunos grupos de escasa relación con la figura: las de gomarresina, del olor del incienso, con brillo de alabastro y otras ${ }^{47}$.

Lo mismo que en la clasificación de las plantas atendiendo a su naturaleza entera, en la consideración de las partes principales también dedica un apartado a la ordenación de las raíces en razón de su comparación con la denominación griega $^{48}$. Aquí, sin embargo, parece más evidente el afán sistemático, porque atiende a distintos tipos morfológicos de raíz, en los cuales pueden englobarse los demás. Son éstos: «Agrosteos», «Bolbi», «Gentianae», «Dauci», «Hellebori», «Iridis», "Calami», "Mandragora», "Panacis», "Raphani», "Scolopendrii», "Teutli», «Hyperici». Más dos que no guardan relación con lo anterior: «radice idem principium sortita» e «inutili radice». Bajo cada epígrafe enumera una serie de grupos de plantas que, de acuerdo con Dioscórides, tienen la raíz similar a la prototípica: mayor, menor, emuladora, etcétera. Por ejemplo, bajo el paraguas de los bulbos cobija tres grupos de plantas, las especies de raíz bulbosa, las especies de raíz a modo de un bulbo grande y las especies de bulbo pequeño.

Ha empezado la descripción de las diferencias dioscorídeas entre las especies del modo habitual, por la raíz. Sigue el tallo o tronco ${ }^{49}$. El primer criterio de comparación, la superficie al tacto del vástago, admite aquí también cierta liberalidad. Incluye la presencia o ausencia (en todo o en parte) de hojas, espinas, lanas, pelos, humores y alguno más sin mayor relación aparente. En cuanto al número, el alumno debe saber que Dioscórides habla de plantas que carecen (siempre o en determinadas circunstancias) de tallo, de plantas con muchos vástagos, de plantas unicaules. En cuanto al tamaño, trae a colación tallos grandes, gruesos, de un dedo de diámetro, finos, largos (con pormenorización de las medidas de longitud), cortos. En cuanto al color, la gama habitual (blancos, lechosos,

\footnotetext{
46 Por ejemplo, «subamara» y "fervida simul et amara». (Stirpium differentiae, p. 1148).

47 «Liquoris gummi referentis... Thuris odore... Similiter alabastris nitente... Clavae alicuius modo nodosa... Pali instar in terram defixa. (Stirpium differentiae, p. 1149).

48 "Radicum inter se collatio iuxta Alphabeti Graeci seriem». (Stirpium differentiae, p. 1149 y ss.).

49 «Plantarum differentiae a caule tractae». (Stirpium differentiae, p. 1151-1158).
} 
azafranados, violáceos, verdes, rojos, negros). En cuanto al sabor, amargos, dulces, aceitosos. En cuanto a la dureza, blandos, tiernos, firmes, duros; ásperos, con variantes y afines. En cuanto a la figura, angulosos, cuadrangulares, geniculados, nudosos, lagunados, etcétera. Y otro cajón de sastre: de tallo arbustivo, leñoso, con genículos a modo de tuba, parecidos al tirso o con figura erecta.

En el apartado que dedica a la memorización de las semejanzas y disimilaridades por razón de la ordenación griega ${ }^{50}$, Textor selecciona con bastante acierto un manojo de prototipos de plantas cuyos tallos sirven de punto de referencia para entender la morfología de otras, de acuerdo con Dioscórides. Indica el nombre griego, añadido de la traducción latina cuando procede; por ejemplo, "Andrachnes. Portulacae», "Ballotes. Marrubii nigri», "Calami. Harundinis", etcétera. Selecciona, por último, otra gavilla de plantas cuyo tallo guardan por una u otra razón parecido, aunque no siempre la comparación sea dos a dos, pese al epígrafe: «Ad binos caules comparatio» ${ }^{51}$.

Tras la raíz y el tallo, los ramos. Se repite el patrón. Superficie (lisos o hirsutos, humores, etcétera), número, tamaño, color, sabor, dureza. Sigue la comparación, a partir de la denominación griega, aunque no lo diga expresamente en el título del epígrafe ${ }^{52}$, de plantas representativas para Dioscórides en razón de los ramos. De acuerdo con el patrón, continúa con el cotejo binario. Pero antes de entrar en el apartado de las hojas introduce una sección que nos revela que Textor asimila la anatomía de la rama a la del tallo, y ambas a estructuras propias de una u otro: «virgae, viticulae, pediculi, culmi, surculi, adnata, germinationes quaedam, festucae, cirri, capreoli». A cada estructura le aplica, en desigual medida, los criterios habituales (superficie, número, talla, etc.) ${ }^{53}$.

$\mathrm{El}$ apartado consagrado a las semejanzas y diferencias de las plantas en razón de las hojas es, con mucho, el más extenso. Abarca de la página 1164 a la página 1182. Empieza, como siempre, con lo que atiende a la superficie foliar (espinosas, aculeadas, hirsutas, tomentosas, etcétera, terminando una vez más con el fluido que exudan), para continuar con el número (especies carentes de hojas y su extremo, estirpes densamente foliadas, adventicias, en los nudos, variables o de cierta cantidad fija); tamaño (grandes, exiguas, gruesas, finas, largas, anchas, oblongas, angostas, con asimiladas y variantes), color, sabor, geometría o forma sensu lato (redonda, acorazonada, angulosa, mucronada, acuminada, nervada, gibosa, cóncava, fisurada, incisa, serrada, dividida, etcétera), figura (en luna, delfín, escolopendra, lengua, pennada, lanceta, cuchillo, acetábulo, huella humana, etcétera).

50 "Caulium inter se affinitas et dissimilitudo secundum Graecarum literarum ordinem». (Stirpium differentiae, p. 1156).

51 (Stirpium differentiae, p. 1158). .

52 "Ramorum inter se comparatio". (Stirpium differentiae, p. 1160-1161).

53 «Stirpium differentiae ab is tractae quae caulium vel ramorum rationem habent." (Stirpium differentiae, , p. 1161-1164). 
En el elenco de voces griegas se muestra mucho más detallista que en los casos anteriores, relativos a la raíz, tallo y ramas. Pudiera estribar la razón de ello en que las hojas constituían, en los comienzos del siglo XVI, el principal criterio de reconocimiento. Este epígrafe podría muy bien constituir un vademécum esencial de la flora que debía conocer el alumno. Apoyado en el Dioscórides, selecciona: "Abrotoni», «Agni. Viticis»; "Agrosteos. Gramini», «Acalyphes. Urticae», "Acanthae leucae. Spinae albae», "Acanthi. Spinae vulgaris», "Amaraci. Sampsuchi», "Ampeli hemerae. Vitis sativae seu cultae», "Ampeli agriae. Vitis sylvestris», "Ampeli leucae. Vitis albae», "Amygdalae», "Anagallidos», "Andrachnes. Portulacae», "Anemones», "Anisi», "Anchusae», "Aparines seu Philantropi», "Aristolocchiae rotundae», "Arnoglossi. Plantaginis», «Atraphaxios. Atriplicis», "Absinthii», "Ballotes. Marrubii nigri», "Batrachii. Ranunculi», «Bolbi. Bulbi», «Buglossi», «Bunii. Napi», «Gingidii», «Glichonis. Pulegii, "Daphnes. Lauri», "Delphinii», "Dracontii. Dracunculi», "Dryos. Quercus», "Elates. Abietis», "Eleae. Oleae», "Elelisphaci. Salviae», "Helxines», «Erebinthi. Ciceris», «Eraces», «Erigerontis. Senecionis», «Herpylli. Serpylli», «Eryngii», «Erythrodani. Rubiae», «Euzomi. Erucae», «Hedyosmi. Mentae», «Heliotropii. Solaris herbae», «Thridacis. Lactucae», «Thymi», "Ipposeline. Oluastri», «Hippuris. Equiseti», «Iridos», «Isatidis. Glasti», «Iteae. Salicis», «Calami. Harundinis»; "Calaminthae. Nepetae», "Cardami. Nasturcii», "Cissi. Hederae», "Cisti», "Cichorii. Ambubeiae», "Cnici. Cartami», "Colocynthae. Cucurbitae», "Conyza», "Corii seu Coriani. Coriandri», "Coronopodis. Cornicis pedis», "Cotyledonis. Acetabuli», "Crambes. Brassicae», "Crites. Hordei», "Crini. Lilii», "Croci», "Cyami Aegyptii. Fabae Aegyptiae», "Cydoniae mali: Cotoneae mali», "Cyclamini. Orbicularis herbae seu rapi terrae», "Cymini sylvestris», "Cyparissi. Cupressi», «Cyperidis», "Cysti», «Conei. Cicutae», «Lapathi. Rumicis», «Lachani. Oleris», «Lepidii», «Libanotidis», «Lini», «Linozosteos. Mercurialis», "Loti pratensis», "Malaches. Malvae», "Mandragorae», "Marathri. Foeniculi», "Melampyri», «Meliloti. Sertulae Campanae», "Melissophylli. Apiastri», "Meconis. Papaveris», "Meleae. Mali», «Moreae. Mori», «Myrsines. Myrti»; "Narthecis. Ferulae», «Xiphii. Gladioli», «Origani», «Panacis», «Pepli», «Periclymeni. Involucri maioris», «Peristereonis. Verbenacae», «Pegani. Rutae», «Pytiós. Piceae», "Platani», «Polypodii. Filiculae», «Prasi. Porri», "Prasii. Marrubii», "Pteridis. Filicis», "Pyxi. Buxi», "Pyri. Tritici», "Rhamni», "Rhodi. Rosae», "Selini. Apii», "Seridis. Intybi», "Sicyi hemeri. Cucumeris sativi», "Sisymbrii», "Scillae», "Scolopendrii. Aspleni», "Scolymi. Cardui», "Scorodi. Allii», "Smyrnii», «Spondylii. Verticilli», «Staphylini. Pastinacae», «Struthii, Radiculae quam proprie Lanariam Latini nominant», "Phyteuma», "Strychni. Solani», "Sices. Ficus», "Schini. Lentisci», "Schoeni. Iunci», "Terminthi. Terebinthi», "Teutli. Betae», "Triphylli. Trifolii», "Hyoscyami. Alterci», "Hyssopi», "Phaci. Lentis», "Phlomi. Verbasci», "Chamaedryos. Querculae», "Chamelaeae. Oleastelli», «Chamaeleonis», "Chamaepityos. Aiugae», "Ocimi». 
Completa ese elenco con un sumario alfabético, griego también, de dieciseis grupos donde se comparan las hojas de una planta con referencia a las de otras. Por ejemplo, bajo el encabezamiento «Sisymbrii» (Nasturtium officinale R. Br.) del epígrafe anterior, Textor asociaba a las hojas de éste las del «Dictamnus altera" (Ballota pseudodictamnus Benth). Ahora, en la "particula decimasexta", recuerda del Dioscórides de Marcello Vergilio que el olor de las hojas del «Dictamnus altera» es suavísimo, medio entre las del sisimbrio y la salvia ${ }^{54}$. Cierra, por fin este capítulo, con tres puntos de interés vario: lugar de inserción de las hojas, tiempo de germinación y carácter urente y escarificante de algunas.

La flor dioscorídea no distingue ninguna función en androceo y gineceo (a esa percepción se llegará a finales del siglo XVII). Se refiere, sobre todo, a la corola. Cuando Textor extraiga de la Materia medica las diferencias entre especies, aludirá a determinadas discrepancias en el perianto. Empezará, de acuerdo con el patrón, con los apéndices espinosos y los humores que despiden. Proseguirá con el número, continuará con el tamaño, color, olor, sabor, dureza, forma y figura. Los prototipos florales, en seriación greco-alfabética, a tenor de los cuales dos plantas se aproximan o distancian (difieren), son: "Anagallidis", "Anemones», «Anethi», «Bolbi. Bulbi», «Helyochrisi», «Ii violae nigrae aut purpurae», «Isatidis. Glasti», "Cichorii. Ambubeiae», "Cambres. Brassicae», "Crinii. Lilii», "Croci», «Cyami. Fabae», «Leucoii. Violae candidae», «Lychnidis», «Meconis. Papaveris», «Narcissi», «Origani», «Pegani. Rutae», «Prasii. Marrubii», "Rhodi. Rosae», "Rhoae. Punicae», "Hyacinthi. Vaccinii», "Hyperici», "Phlomi. Verbasci», "Chamaemali. Anthemidis», «Ocimi». Sin solución de continuidad, es decir, sin introducir el epígrafe donde procede a comparar dos o más especies con otra en razón de la flor, recoge la semejanza entre la flor de "Cacalia» con la del «Muicus» y «Olea». De acuerdo con Dioscórides retiene que la flor puede brotar en distintas partes de la planta: arrancar en la misma base, al final del vástago, en ramas. Confunde la estructura de soporte de la flor. Por último, señala el tiempo de floración de algunas plantas y el carácter urticante y ulcerante de todos los ranúnculos.

Entre las escasas anotaciones personales de Textor, no extraídas directamente de la Materia medica, sobresale la que aparece en este apartado de la flor. Dice allí que el fruto, la semilla, las «umbellae» y los "pericarpia» siguen necesariamente a la flor. Por eso, agrega, deben hallarse cerca de la misma. No siempre ocurre así con los «capitula» 55 .

\footnotetext{
54 «Inter Sisymbrium et Elelisphacum, medium odorem qui suavissimus est, fragrante, Dictamnus altera». (Stirpium differentiae, p. 1181).

55 «Fructus, seminis et caeterorum quae florem necessario consequuntur, ut umbellarum et pericarpiorum, perspicuum est eandem prope cum illo esse positionem. Capitulorum tamen, cum florem impsum non semper respiciant, situs non erat huc referentibus.) (Stirpium differentiae, p. 1188).
} 
Distingue entre fruto y semilla, como si se tratara de dos elementos separables, aunque relacionados. Puede haber frutos sin endocarpo que encierren las semillas, como en el caso del madroño ${ }^{56}$. Las diferencias entre especies en razón del fruto empiezan por los detalles externos o superficiales, con algún rasgo más sin vinculación con ese criterio (jugo que rezuma). Pasa luego al número, desde las que carecen de fruto («steriles») hasta los que los poseen en abundancia, el tamaño, color, olor, sabor, textura, forma geométrica y figura. Los tipos de frutos, es decir, las especies que portan frutos que sirven, en Dioscórides, de referencia para otros son: "Anethi», "Amygdalae», "Balani. Glandis», «Botryos et Staphyles. Racemi et uvae», «Elaeae. Olivae», «Erinei. Caprifici», «Thermi. Lupini», "Caryi basilici. Nucis regiae», "Capparis», "Coccimilei. Pruni", "Corymbi. Baccae Hederaceae», "Coni seu Strobili. Pineae nucis», «Libanotidis», «Mandragorae foeminae», "Meconis ceratitis. Papaveris cornuti», «Myrti», «Olynthorum. Grossulorum», «Pepereos. Piperis», «Platani», «Pyri. Tritici», «Rhagis staphyline. Acinis uvacei». «Sicyi hemeri. Cucumeris sativi», "Syces. Ficus», «Schini. Lentisci» y "Terminthi. Terebinthi». Por último, una observación sobre el boj ("pendulo fructu») y frecuencia de fructificación.

La clasificación de base dioscorídea que Textor realiza de las plantas en razón de la semilla se extiende desde la página 1191 hasta la 1197. De acuerdo con el patrón, arranca de la superficie al tacto (tomentosidad por ejemplo), con mezcla de otras peculiaridades, aquí de particular interés, como la ambrosía, que produce semillas sin flor previa, o la parietaria, cuasi dotada de semillas, amén del acostumbrado fluido exudante. Y, a continuación, las notas accidentales de rigor: número, tamaño, color, olor, sabor, textura, forma geométrica, figura. Las plantas cuyas semillas sirven de referencia en Dioscórides, ya sea por semejanza o disparidad ${ }^{57}$, son las siguientes: "Anchusae", "Altheae, "Ammi», "Anethi», "Anisi», "Apii. Piri», «Asphodeli. Hastulae regiae», "Atraphaxios. Atriplicis», "Absinthii», "Botryos. Racemi», «Gigartorum. Nucleorum uvae», "Gliconis. Pulegii», «Erebinthi. Ciceris», «Erysimi. Irionis», «Hedysari. Securidacae», "Canchryos. Seminis Libanotidis», «Cardami. Nasturtii», Cenchri. Milii», "Cissi. Hederae», "Cnici. Cartami», "Corymbi. Baccae Hederae», "Crambes. Brassicae», «Crithes. Hordei», "Cyami. Fabae», "Cymini», «Lini», "Marathri. Foeniculi», Melanthii. Git sive Nigellae», "Meconis. Papaveris", "Myrsines. Myrti», «Narthecis. Ferulae», «Origani», «Ormini», «Orobi. Ervi», "Oxyschoeni. Acuminati Iunci», «Peperos. Piperi», «Prasi. Porri», «Pyri. Tritici», "Rhoae. Punicae», "Selini. Apii», "Sesami»,»Spondylii. Verticillaris. Herbae», "Schini. Lentisci», «Teutli. Betae», «Teleos. Foeni graeci», «Tithymali characiae. Lactaria vallaris», «Hyoscyami. Alterci», «Hyperici» y «Phaci. Lentis». Para ter-

56 "Nucleis vacuo, Comaros, id est, Arbutus». (Stirpium differentiae, p.1188).

57 «Seminum inter se aemulatio et diversitas». (Stirpium differentiae, p. 1195-1197). 
minar con algunas variaciones sobre el fruto donde se encierran las semillas («in calyculis», «in pomis», «membrana incluso», otra sobre la estación de maduración y una última sobre la planta de semillas vanas ${ }^{58}$.

Resume, por fin, en ocho apartados otras estructuras anatómicas ligadas a la flor, el fruto y la semilla, en razón de las cuales las especies se asemejan o discrepan. Esas estructuras no se corresponden forzosamente con las designadas actualmente por esos mismos nombres: cáliz, verticilo, espiga, umbela, capítulos y vasos, silicuas, papos y espinas ${ }^{59}$. Se trata de destacar aquellas plantas que portan de una manera llamativa ese rasgo, según Dioscórides. Del cáliz, por ejemplo, registra el de la barba de chivo (Tragopogon porrifolius L.); de las espigas, la del raigrás (Lolium perenne L.), entre otras; de las portadoras de umbela, la de la cicuta (Conium maculatum L.), entre varias; del vilano, "Typha» (Typha angustifolia L. y T. latifolia L.); de la espina, el cardo (Cynara cardunculus L.) y algunas más. Por lo que respecto a capítulos y vasos, entendiendo por tales formaciones foliares en copa, más que estructuras de disposición de la flor, remeda el patrón habitual seguido en raíces, tallos, etcétera: presencia o ausencia y sensibilidad al tacto, número, color, olor, sabor, forma geométrica y figura que imita. A ello añade la comparación, binaria o no, entre plantas partiendo de la denominación griega, para continuar con el punto de aparición o inserción del «caput». También se aplica el patrón general a las silicuas.

La intención de Silvio, de Textor, es que los futuros médicos, los «tyrones», conozcan de una forma sistemática las propiedades galénicas de las plantas, de las principales al menos, en lo que se refiere al sabor, olor, textura de las partes $\mathrm{y}$ otras indicaciones necesarias ${ }^{60}$. Puesto que en esos años del primer tercio del XVI el esfuerzo de los naturalistas se centraba en recuperar el significado exacto de las plantas descritas por Dioscórides, y apenas si había empezado a incrementarse su elenco, nada mejor que disponer de una guía que permitiera memorizar y extraer el mayor aprovechamiento del nuevo canon. Y hacerlo, además, científicamente, aplicando para su sistematización las herramientas de la lógica aristotélica. Otras obras, con propósito similar, le seguirán, impulsadas por el propio Silvio. Pero eso nos aparta de los límites de nuestro ensayo.

\footnotetext{
58 "Inutile semine, Poterium» (Asytagalus poterium Labill.). (Stirpium differentiae, p. 1197).

59 Stirpium differentiae, p. 1197-1200).

60 «Itaque hortor omnes rei medicae studiosos, praecipues tyrones, ut stirpium omnium, aut saltem praecipuarum et quae in aliquo usu receptae sunt, temperamenta, excessus et qualitates perdiscant, cum ex veterum libris, ubi et librarii multa corruperunt et authores ipsi aliquoties aberrarunt, tum ex universali quadam methodo et scientia, quae fere ex sapore, odore, colore et partium crassitie aut tenuitate deducitur, et aliis quibusdam indicis ratione vel experientia cognoscendi, etc." (Gesnerus, $C$.: "De illis qui de medicamentorum simplicium facultatibus secundum differentias qualitatum primarum, secundarum, et reliquarum scripserunt», l.c. s.p.).
} 